\title{
Fatores de risco e mortalidade em pacientes criticamente enfermos com infecções por microrganismos multirresistentes
}

\author{
Risk factors and mortality in critically ill patients with infections with multidrug-resistant \\ microorganisms
}

\author{
Factores de riesgo y mortalidad en pacientes críticos con infecciones por \\ microorganismos multirresistentes
}

Luzia Franciny de Souza Pinheiro ${ }^{1 *}$, Carolina Palmeira Teixeira Martins ${ }^{2}$, Clézio Figueiredo Martins ${ }^{3}$, Poliana Terra Pires Ribeiro Coelho Caires ${ }^{1}$, Vitor Santos Aragão ${ }^{1}$, Otilia Santos Aragão ${ }^{1}$, Ramon Sales Ferraz ${ }^{1}$, Yasmin dos Santos Felisberto ${ }^{1}$, Francisco José Liberato de Mattos Carvalho Neto ${ }^{1}$, Mariane Cardoso Chaves ${ }^{1}$.

\section{RESUMO}

Objetivo: Revisar e analisar os principais fatores de risco que predispõe as infecções por microrganismos multirresistentes em pacientes criticamente enfermos, relacionando-os com a mortalidade, bem como avaliar a prevenção dos mesmos. Revisão bibliográfica: As infecções causadas por patógenos multirresistentes é um problema significativo na prática médica e está associada à cenários em que os indivíduos são submetidos a uma conduta terapêuticas e linha de cuidados prolongados. Nessa perspectiva, os pacientes em estado crítico tornam-se vulneráveis e se inserem cada vez mais nos números crescentes de morbimortalidade associados à resistência antimicrobiana, sendo esta promovida principalmente por bactérias gram-negativas. Além do uso indiscriminado desse medicamento, outros fatores como idade avançada, sexo masculino, imunocomprometimento e comorbidades estão associados a esse fenômeno. Considerações finais: Uma maior conscientização sobre a multirresistência bem como a importância da sua prevenção e controle de fatores de riscos individuais e ambientais que podem desencadeá-la pode ajudar a reduzir a morbidade e mortalidade de pacientes criticamente enfermos, os quais estão mais expostos a esse problema. Intervenções eficazes como estreitar o uso de antibióticos diante de resultados diagnósticos, desenvolver práticas de controle de infecção em ambiente hospitalar, promovem o seu uso criterioso e evita consequências prejudiciais decorrentes da multirresistência.

Palavras-chave: Multirresistentes, Microrganismos, Pacientes, Mortalidade, Fatores de risco.

\begin{abstract}
Objective: To review and analyze the main risk factors that predispose infections by multidrug-resistant microorganisms in critically ill patients, relating them to mortality, as well as assessing their prevention. Bibliographic review: Infections caused by multidrug-resistant pathogens is a significant problem in medical practice and is associated with scenarios in which individuals are subjected to therapeutic conduct and longterm care. In this perspective, critically ill patients become vulnerable and are increasingly inserted in the increasing numbers of morbimortality associated with antimicrobial resistance, which is mainly promoted by gram-negative bacteria. In addition to the indiscriminate use of this medication, other factors such as advanced age, male gender, immunocompromise and comorbidities are associated with this phenomenon. Final considerations: Increased awareness of multidrug resistance as well as the importance of its prevention and control of individual and environmental risk factors that can trigger it can help to reduce the morbidity and mortality of critically ill patients, who are more exposed to this problem. Effective interventions such as narrowing the use of antibiotics in the face of diagnostic results, developing infection control practices in the hospital environment, promoting their judicious use and avoiding harmful consequences resulting from multidrug resistance.
\end{abstract}

Keywords: Multidrug resistant, Microorganisms, Patients, Mortality, Risck factors.

${ }^{1}$ Faculdades Santo Agostinho (FASA), Vitória da Conquista - BA. *E-mail: francinypinheiro@hotmail.com

${ }^{2}$ Centro Universitário Serra dos Órgãos (UNIFESO), Teresópolis - RJ.

${ }^{3}$ Escola Bahiana de Medicina e Saúde Pública (EBMSP), Salvador - BA.

SUBMETIDO EM: 4/2021

ACEITO EM: 4/2021

PUBLICADO EM: 4/2021 


\section{RESUMEN}

Objetivo: Revisar y analizar los principales factores de riesgo que predisponen a infecciones por microorganismos multirresistentes en pacientes críticos, relacionándolos con la mortalidad, así como evaluando su prevención. Revisión bibliográfica: Las infecciones causadas por patógenos multirresistentes constituyen un problema significativo en la práctica médica y están asociadas a escenarios en los que los individuos son sometidos a conductas terapéuticas y cuidados a largo plazo. En esta perspectiva, los pacientes en estado crítico se vuelven vulnerables y se insertan cada vez más en el creciente número de morbimortalidad asociadas con la resistencia a los antimicrobianos, promovida principalmente por bacterias gramnegativas. Además del uso indiscriminado de este medicamento, otros factores como edad avanzada, sexo masculino, inmunodepresión y comorbilidades se asocian a este fenómeno. Consideraciones finales: Una mayor conciencia de la multirresistencia así como la importancia de su prevención y control de los factores de riesgo individuales y ambientales que pueden desencadenarla puede ayudar a reducir la morbilidad y mortalidad de los pacientes críticos, que están más expuestos a este problema. Intervenciones eficaces como la reducción del uso de antibióticos ante los resultados del diagnóstico, el desarrollo de prácticas de control de infecciones en el entorno hospitalario, la promoción de su uso prudente y la prevención de las consecuencias nocivas derivadas de la multirresistencia.

Palabras clave: Multirresistente, Microorganismos, Pacientes, Mortalidad, Factores de riesgo.

\section{INTRODUÇÃO}

As bactérias são organismos diversos que variam tanto em sua forma quanto em sua relação com seu hospedeiro, podendo apresentar um comportamento patogênico. Assim, quando esse microrganismo oferece uma ameaça à saúde do indivíduo há a necessidade de utilizar antimicrobianos, contudo, a resistência a esses agentes está se tornando um problema cada vez mais atual no cenário científico (JAMESON JL, et al., 2020). Apesar desse fenômeno ocorrer em decorrência de uma interação natural entre o organismo e o meio ambiente em que está inserido, estudos europeus alertam sobre o aumento da incidência desse comportamento bacteriano nos últimos anos, revelando que $67,6 \%$ das mortes decorrentes de infecção são provocadas por bactérias classificadas como multirresistentes à antibióticos (SILVA RA, et al., 2020).

De uma maneira geral, uma bactéria é considerada resistente a um antibiótico quando apresenta uma resistência in vitro a uma concentração inibitória do fármaco. Esse efeito pode é considerado simples quando é apenas a uma droga ou múltiplo quando não há suscetibilidade simultânea a duas ou mais (TAVARES W, 2020). Nesse cenário, existem vários fatores comportamentais que contribuem para que esses microrganismos não sejam mais suscetíveis, como o alto consumo e uso inadequado dos antibióticos, a utilização excessiva de antimicrobianos na pecuária, causando um efeito indireto para os humanos, a poluição ambiental associada ao descarte incorreto desses fármacos no solo ou na água e as deficiências regulamentares que compõem as diretrizes de fiscalização desses medicamentos (KAAE S, et al., 2017).

Diante disso, o microrganismo pode adquirir a capacidade de opor-se aos efeitos inibidores do crescimento ou pode resistir ao efeito bactericida de uma substância antimicrobiana. Existe uma distinção entre a resistência primária, que é uma espécie ou característica genérica, e a resistência secundária adquirida, que é a base da multirresistência. Uma espécie de bactéria pode se tornar resistente através da alteração da estrutura das moléculas alvo dos antibióticos, pelo desenvolvimento de mecanismos de bomba que levam ao efluxo desse medicamento da célula e pela produção de enzimas que inativam tal fármaco, o que acarreta na redução da penetração desse antimicrobiano na célula bacteriana (HAGEL S, et al., 2015).

Debater sobre esse tema é importante, pois as infecções causadas por patógenos multirresistentes é um problema crescente na prática médica e está associada à cenários em que os pacientes são submetidos a uma conduta terapêuticas e linha de cuidados prolongados (BARROS FE, et al., 2019). Nessa perspectiva, temos que as infecções resistentes à antibióticos, principalmente aquelas causadas por Organismos Multidroga Resistentes (MDROs), representam uma grande ameaça à saúde pública global. Tal fato merece uma atenção especial, pois os pacientes criticamente enfermos com exposição prévia a antimicrobianos estão mais suscetíveis a adquirir infecção por MDROs, o que aumenta os custos de hospitalização, as taxas de mortalidade e o tempo de internação hospitalar (GANDRA S, et al., 2019). 
Corroborando com essa perspectiva, Matos ECO, et al. (2018) em uma pesquisa de metanálise revelou que além dos altos custos que as infecções associadas aos MDROs acabam gerando, a complexidade dos casos também aumenta. Proporcionalmente, a necessidade em que os indivíduos adquirem em permanecer por longos períodos hospitalizados para realizarem o tratamento adequado os deixam mais vulneráveis às bactérias de difícil erradicação e controle. Logo, consequentemente, todo esse processo contribui para a ocorrência de desfechos desfavoráveis. Assim, quando se compara pacientes infectados por patógenos resistentes em detrimento aos não resistentes a taxa de mortalidade aumenta significativamente no primeiro grupo.

Compreender todas essas questões dentro do ambiente hospitalar no qual os pacientes criticamente enfermos procuram tratamento é essencial para manejar esse desafio. Afinal, a resistência antimicrobiana também está atrelada ao uso excessivo de antibióticos, prescritos por profissionais de saúde, de maneira empírica e sem a realização de um antibiograma para posterior ajuste no tratamento. Dessa forma, utilizamse, muitas vezes, drogas pré-estabelecidas e desnecessárias para a verdadeiro quadro clínico do paciente. Além disso, existe a falta de conhecimento sobre o estágio da infecção e dos diversos organismos existentes bem como a falta de controle dos serviços de saúde sobre os processos básicos de higienização, como a lavagem correta das mãos, potencializando a existência de infecções cruzadas (WATTAL C, et al., 2019).

Portanto, partindo desses pressupostos e considerando a relevância desse tema dentro do âmbito da saúde pública, esse estudo possui como objetivo revisar e analisar os principais fatores de risco que predispõe as infecções por microrganismos multirresistentes em pacientes criticamente enfermos, relacionando-os com a mortalidade, bem como avaliar a prevenção dos mesmos.

\section{REVISÃO BIBLIOGRÁFICA}

Antimicrobiano é toda aquela substância, de origem natural ou sintética, que age sobre microrganismos podendo controlar o seu crescimento ou até mesmo provocando a sua aniquilação. Rotineiramente usados dentro de estabelecimentos ambulatoriais ou hospitalares de modo profilático e terapêutico, esses fármacos possuem uma importância significativa no avanço de diversos tratamentos de patologias. Porém, sua utilização não criteriosa e amplamente indiscriminada contribui para a ocorrência da resistência bacteriana e de suas consequências, como a potencialização de cepas de difícil controle, piora significativa no quadro clínico de pacientes críticos inseridos no ambiente hospitalar e oneração dos planos terapêuticos (FURTADO DMFF, et al., 2019).

À vista disso, diversos estudos inseridos na comunidade científica vêm sugerindo uma associação entre o uso não racional de antibióticos, o aumento da resistência antimicrobiana e elevação da morbimortalidade em pacientes hospitalizados em estado crítico (CABRAL LG, et al., 2018). Segundo Borgatta B, et al. (2018), na última década, por exemplo, houve um aumento nas cepas de Pseudomonas MDR nas Unidades de Terapia Intensiva (UTI) e os pacientes internados nesse ambiente, além de gravemente debilitados, apresentaram problemas coexistentes, marcadores inflamatórios elevados e foram frequentemente expostos a antibioticoterapia prévia desnecessária. Logo, esse cenário dificulta o manejo e contribui como fator de risco para o desenvolvimento da resistência desse microrganismo.

Nessa perspectiva, temos que o surgimento e disseminação da $E$. coli produtora de $\beta$-lactamase cresceu e é relatada globalmente. Bala R, et al. (2020) evidenciou em sua pesquisa que as principais condições associadas a presença de E.coli MDR isolada em várias amostras clínicas de pacientes hospitalizados foram, respectivamente: uso prévio de antibióticos $(90 \%)$, estado pós-operatório $(65,4 \%)$, permanência hospitalar prolongada $(53,8 \%)$, uso de cateter urinário (52\%), idade maior que 60 anos $(46,1 \%)$ e imunocomprometimento (17,3\%). Com isso, observa-se que tanto os aspectos inerentes ao próprio indivíduo, como a idade e o estado do sistema imunológico, quanto o uso de procedimentos invasivos e condições préestabelecidas influenciam na predisposição de desenvolver infecções resistentes.

Dessa forma, há uma preocupação pertinente na comunidade científica no que discerne a eficiência dos fármacos que estão dispostos no mercado. Foi demostrado que as infecções causadas por Acinetobacter MDR eram de difícil tratamento devido à pouca disponibilidade de antibióticos eficazes. Esse fato confirma a 
atual mudança na prevalência de MDROs gram-positivos para gram-negativos devido à falta de agentes efetivos para combater cepas gram-negativas resistentes. A carência de medicamentos contra esses tipos de patógeno tem se tornado um preocupante fator de risco para a população, pois favorece a perpetuação da multirresistência em ambientes clínicos (FRANCESCO MA, et al., 2013).

Os pacientes expostos empiricamente as principais classes de antibióticos apresentam maior risco de desenvolver infecções por MDROs. O uso prévio desses medicamentos favorece a pressão seletiva bacteriana, pois produz mecanismos de resistência e dificulta o tratamento efetivo (LIU P, et al., 2018). Além disso, aqueles pacientes quando acometidos por infecções MDR originadas a partir de bacilos gram-negativos apresentam uma maior taxa de mortalidade quando associados a diabetes mellitus, doença hepática em estado terminal, câncer em órgãos sólidos, uso de cateter urinário, infecções intra-abdominais e submissão de terapias empíricas prévias inadequadas (PATOLIA S, et al., 2018).

Todavia, o próprio cenário de multirresistência não influencia apenas nas pessoas com comorbidades, mas também na própria existência e tipo de infecção, como ocorre nas que acometem a corrente sanguínea. As taxas de mortalidade nesses casos são altíssimas, variando entre $40 \%$ a $60 \%$ nos pacientes que estão inseridos dentro de UTIs. Assim, os indivíduos hospitalizados que adquiriram infecções em decorrência da proliferação de bactérias classificadas como extremamente multirresistentes normalmente pertenciam ao grupo de gram-negativas e possuíam um risco maior de ir ao óbito, independente da condição clínica ou comorbidade prévia (SANTORO A, et al., 2020).

Um estudo feito por Gandra S, et al. (2019) mostrou que entre as infecções por microrganismos multirresistentes, os patógenos gram-negativos mais prevalentes associados a mortalidade de pacientes criticamente enfermos foram: Escherichia coli, Klebsiella pneumoniae, Pseudomonas aeruginosa e Acinetobacter baumannii. Por outro lado, os patógenos gram-positivos mais prevalentes foram: Staphylococcus aureus e Enterococcus spp. Entre esses pacientes a taxa de letalidade por infecções MDRPs foram mais altas entre as causadas por bactérias gram-negativas $(17,7 \%)$, em oposição às causadas por bactérias gram-positivas (10,8\%). Assim, nota-se mais uma vez como os microrganismos resistentes influenciam no aumento da mortalidade.

Diante desses fatos, temos que epidemiologicamente no continente europeu morrem 25.000 pessoas todos os anos em virtude de infecções bacterianas multirresistentes. Para mais, os dados referentes aos Estados Unidos da América (EUA) revelam que mais de 2 milhões de pessoas são infectadas com MDR, refletindo em 23.000 mortes diretas (BLAIR JM, et al., 2015). Assim, temos que a resistência aos antimicrobianos aumenta em aproximadamente 700.000 o número de mortes anualmente (UC-CACHÓN AH, et al., 2019) e estima-se que em 2050 esse número aumente de forma alarmante para 10.000.000 (KRAKER $\mathrm{ME}$, et al., 2016).

Partindo desses pressupostos, temos que as infecções relacionadas aos MDROs possuem um significado clínico muito relevante, principalmente sobre os desfechos de pacientes internados em UTI, pois são os locais de considerável prevalência de infecções resistentes devido a gravidade e a vulnerabilidade aos quais esse grupo de indivíduos estão expostos (UC-CACHÓN AH, et al., 2019). Na região norte americana, 1 em cada 25 pacientes internados em hospitais de cuidados agudos tem pelo menos uma infecção associada aos cuidados de saúde (MAGIL SS, et al., 2014). Entre as infecções mais comuns adquiridas na UTI estão a pneumonia, infecção do sítio cirúrgico, infecção gastrointestinal, infecção do trato urinário e infecção da corrente sanguínea (MACVANE SH, et al., 2017).

Pesquisas em diversos países apontam que fatores de risco que predispõe infecções bacterianas resistentes envolvem tempo de permanência em unidades de cuidados antes da infecção, exposição a antibióticos nas últimas seis semanas, retorno recente de viagens ao exterior, admissão em UTI, residência em instituição de longa permanência, sexo masculino, idade mais avançada e presença de comorbidades. Esses fatores foram tidos como sendo independentes para o desenvolvimento de resistência. A identificação desses aspectos se mostrou crucial no combate as infecções por MDR, pois garante uma terapia oportuna e congruente em pacientes de maior risco (OSTHOFF M, et al., 2015). 
Os fatores individuais para aquisição de MDROs são o tempo de internação hospitalar prolongado, a exposição aos procedimentos invasivos, como a ventilação mecânica, o uso de agentes antimicrobianos de amplo espectro, cirurgia recente e a gravidade da doença subjacente. É necessário considerar também o local onde os pacientes criticamente enfermos se encontram devido ao risco da presença de MDROs já preexistentes no ambiente, pois pode ser fonte de contaminação para indivíduos ainda não infectados por esses microrganismos. Junto às condições externas, deve-se também considerar o contato entre os pacientes e os profissionais de saúde, pois o controle e cuidado com a higienização deve ser destacado a fim de se evitar a disseminação desses microrganismos nos serviços (KERNÉIS S e LUCET JC, 2019).

Ao analisarmos aqueles pacientes inseridos dentro da faixa etária pediátrica, temos que os fatores de risco apontado em adultos também se assemelham para esse grupo de indivíduos. Um estudo retrospectivo realizado em crianças na Guatemala em 2017 mostrou que as infecções com MDR em pacientes pediátricos oncológicos tem sido causa frequente de morbidade e mortalidade, no qual os fatores de risco mais relevantes para o desenvolvimento dessa enfermidade são: idades mais avançadas e infeções tratadas anteriormente com quatro ou mais antibióticos. Portanto, nota-se que mesmo em crianças que apresentem doenças de base, o uso sem critérios de antimicrobianos para tratar infecções pode ser danoso e influenciar diretamente na recuperação e no desfecho dos pacientes (SANDOVAL P e CHANG A, 2017).

Entretanto, a atenção do profissional de saúde não deve se restringir apenas na identificação dos fatores que predispõe essas condições, mas também nas principais complicações presentes nesse perfil de pacientes hospitalizados e criticamente enfermos. À vista disso, um levantamento feito em um centro de pesquisa de cuidados terciários observou que a pneumonia associada a ventilação (PAV) é uma importante complicação responsável pelo aumento da morbidade e mortalidade nesse cenário. Todavia, apesar dos patógenos relacionados a PAV apresentarem diferenças quanto a sua virulência e potencial patogênico, frequentemente eles se apresentam como multirresistentes e devem ser alvo de uma atenção, pois oferece um risco de vida seis vezes mais preocupante quando comparado com os pacientes sem MDRO (TEDJA R, et al., 2014).

Nesse contexto, a promoção de uma conduta responsável e consciente do médico torna-se fundamental, pois a prescrição adequada e, consequentemente, o uso prudente dos fármacos pode fazer com que a crescente resistência antimicrobiana diminua (HARA GL, et al., 2016). A administração apropriada desses medicamentos deve ser idealmente baseada em critérios e evidencias clínicas. Com isso, há uma otimização no tratamento das doenças e uma redução na obtenção e disseminação de bactérias MDR. Afinal, saber dos padrões de resistência local e dos fatores de riscos individuais que predispõem a resistência acarreta em um melhor suporte de atendimento em pacientes criticamente enfermos (MORRIS S e CEREO E, 2020).

O aumento da prevalência de MDR é ainda um desafio para os médicos que lidam com pacientes em internados em estado crítico, pois uma possível infecção por esses microrganismos aumenta a probabilidade de falha na terapêutica inicial. O uso da terapia de amplo espectro na tentativa primária de conter essas infecções tem grandes chances de não surtir o efeito desejado e ainda aumentar a resistência. Neste sentido, torna-se mandatório a realização prévia de exames como culturas e antibiograma nos pacientes para o tratamento adequado, porém essa não é a realidade na maioria dos centros de saúde em tempo hábil e o tratamento é iniciado antes dos testes serem realizados (PILTCHER OB, et al., 2018; CABRAL LG, et al., 2018).

Dessa forma, percebe-se que a resistência antimicrobiana tem origem multifatorial. Dentre os fatores que predispõe essas infecções o que mais se encontra é falta da correta administração de antibióticos, o que leva ao seu uso excessivo, ampla cobertura empírica inadequada e atrasos na precisão dos diagnósticos. Além disso, a desaceleração da terapêutica se inclui na raiz do problema visto que estudos apontam que é preciso reavaliar as prescrições desses fármacos num determinado período de tempo para que se observe uma evolução clínica do quadro infeccioso, ao que se obtêm os resultados de culturas para, então, fazer uma reabordagem no intuído de mudar ou não o esquema de tratamento (MORRIS S e CEREO E, 2020; PULCINI C, et al., 2008).

No que se discute sobre a necessidade do tratamento inicial, é preciso ter cuidado e fazer algumas considerações antes que a terapia seja instituída: considerar a doença subjacente bem como sua gravidade 
e os possíveis patógenos com potencial de colonização; identificar se o paciente teve alguma infecção anterior que possa aumentar a probabilidade de resistência; analisar a microbiota bacteriana e as tendências a resistência dessas no ambiente hospitalar; e por fim, administrar a terapia antimicrobiana empírica tendo como base essas considerações até que esse regime terapêutico possa ser substituído frente aos resultados do antibiograma. (KOLLEF MH, et al., 2011).

Apesar de muitas diretrizes indicarem o uso de terapia empírica frente a uma infecção bacteriana, esta deve ser usada de forma consciente e deve ser considerado o potencial de resistência frente aos patógenos mais comuns diante das condições críticas apresentadas pelo paciente (RAMAN G, et al., 2015). Somado a isso, é preciso fazer a identificação adequada do patógeno multirresistente através de culturas e teste de suscetibilidade. A dosagem e a duração do tratamento também devem ser levadas em consideração para garantir uma cobertura eficiente e exposição (MORRIS S e CEREO E, 2020).

Nessa perspectiva, o descalonamento da terapia surge como uma estratégia para racionalizar o uso do antimicrobiano. Assim, o profissional de saúde deve agir por etapas, administrando inicialmente o tratamento empírico de amplo espectro quando necessário e ajustar a conduta terapêutica, como alteração de terapia combinada para monoterapia ou até mesmo suspensão do fármaco, assim que o patógeno for identificado. Todo esse processo contribui para a redução da morbimortalidade dos pacientes em estado crítico, principalmente naqueles que tiveram como complicação a PAV (CABRAL LG, et al., 2018).

Considerando todos esses pressupostos e a importância do profissional de saúde nesse cenário, o acompanhamento desses pacientes por especialistas em infectologia torna-se uma estratégia importante para um bom prognóstico, pois esses médicos possuem um cuidado maior no controle do uso deliberado de antibióticos de amplo espectro. De acordo com Kim I, et al. (2018) a presença de infectologistas gera um impacto na redução da mortalidade associada a infecções por microrganismos gram-negativos, pois seu conhecimento específico auxilia tanto no acompanhamento do paciente quanto no seu manejo eficiente, reduzindo assim a mortalidade intra-hospitalar e melhorando o desfecho de indivíduos criticamente enfermos.

Como visto, muito fatores contribuem para o aumento da prevalência de bactérias multirresistentes. Fatores esses que acabam por perpetuar o ciclo de aumento de resistência, gravidade dos pacientes e morte, dificultado uma efetiva redutibilidade do problema. A falta de uma administração consciente de antimicrobianos de amplo espectro assim como a falta de boas práticas de controle dessas infecções acabam por dificultar o cuidado adequado dessas infecções, levando a prognósticos desfavoráveis dos pacientes. (CEREO E, et al., 2016). Assim, temos como principal estratégia a educação permanente nos profissionais de saúde e a promoção e compartilhamento de informações sobre esse tema nos ambientes comunitários a fim de oferecer uma conscientização plena (SALDANHA DMS, et al., 2018).

Portanto, a resistência aos antibióticos é um problema de saúde a ser enfrentado pelos próximos anos, considerando que poucos avanços foram alcançados no sentido de produção de novos medicamentos que venham a combater organismos multirresistentes. Para mais, deve-se promover a criação e consolidação de medidas de prevenção e controle de fatores que facilitam as infecções contra esses patógenos resistentes. Além disso, é preciso chamar atenção para alternativas promissoras que podem, futuramente, contornar esse problema como a nanoterapia que, em estudos, já demonstram comprovação na otimização de terapias já existentes (VIVAS R, et al., 2019).

\section{CONSIDERAÇÕES FINAIS}

A resistência a antibióticos e o crescimento de cepas de organismos resistentes representa uma situação de emergência em saúde por serem responsáveis por altas taxas de mortalidade e deixarem poucas opões de tratamentos eficazes na atualidade. Assim uma maior conscientização sobre a multirresistência, bem como a importância da sua prevenção e controle de fatores de riscos individuais e ambientais que podem desencadeá-la pode ajudar a reduzir a morbidade e mortalidade de pacientes criticamente enfermos, os quais estão mais expostos a esse problema. O estudo sobre essa temática se faz necessário para desenvolver e organizar intervenções apropriadas, principalmente, no que diz respeito ao tratamento empírico inicial com antimicrobianos de amplo espectro. Intervenções eficazes, como estreitar o uso de antibióticos diante de resultados diagnósticos, desenvolver práticas de controle de infecções em ambientes. 


\section{REFERÊNCIAS}

1. BALA R, et al. Prevalence, multidrug-resistance and risk factors for AmpC $\beta$-lactamases producing Escherichia coli from hospitalized patients. J Infect Dev Ctries., 2020; 14(12):1466-1469.

2. BARROS FE, et al. Controle De Infecções A Pacientes Em Precaução De Contato. Revista de Enfermagem UFPE, 2019; 13(4): 1081-1089.

3. BLAIR JM, et al. Molecular mechanisms of antibiotic resistance. Nat Rev Microbiol., 2015; 13(1): 42-51.

4. BORGATTA B, et al. Infections in intensive care unit adult patients harboring multidrug-resistant Pseudomonas aeruginosa: implications for prevention and therapy. Eur J Clin Microbiol Infect Dis., 2017; 36(7): 1097-1104.

5. CABRAL LG, et al. Racionalização de antimicrobianos em ambiente hospitalar. Rev Soc Bras Clin Med., 2018;16(1): 59-63.

6. CERCEO E, et al. Multidrug-Resistant Gram-Negative Bacterial Infections in the Hospital Setting: Overview, Implications for Clinical Practice, and Emerging Treatment Options. Microb Drug Resist., 2016; 22(5): 412-31.

7. FRANCESCO MA, et al. Prevalence of multidrug-resistant Acinetobacter baumannii and Pseudomonas aeruginosa in an Italian hospital. J Infect Public Health, 2013; 6(3): 179-85.

8. FURTADO DMFF, et al. Consumo de antimicrobianos e o impacto na resistência bacteriana em um hospital público do estado do Pará, Brasil, de 2012 a 2016. Rev Pan-Amaz Saude, 2019; 10: e201900041.

9. GANDRA S, et al. The Mortality Burden of Multidrug-resistant Pathogens in India: A Retrospective, Observational Study. Clin Infect Dis., 2019; 69(4): 563-570.

10. HAGEL S, et al. Multiresistente Erreger [Multiresistant Organisms]. Zentralbl Chir., 2015; 140(4): 417-25.

11. HARA GL, et al. Ten key points for the appropriate use of antibiotics in hospitalised patients: a consensus from the Antimicrobial Stewardship and Resistance Working Groups of the International Society of Chemotherapy. Int $\mathrm{J}$ Antimicrob Agents, 2016; 48(3): 239-46.

12. JAMESON JL, et al. Medicina interna de Harrison. 20a. ed. Porto Alegre: AMGH, 2020; 13357p.

13. KAAE S, et al. Antibiotic knowledge, attitudes and behaviours of Albanian health care professionals and patients-a qualitative interview study. J Pharm Policy Pract., 2017; 10(13):1-8.

14. KERNÉIS S, LUCET JC. Controlling the Diffusion of Multidrug-Resistant Organisms in Intensive Care Units. Semin Respir Crit Care Med., 2019; 40(4): 558-568.

15. KIM I, et al. Current Status and Survival Impact of Infectious Disease Consultation for Multidrug-Resistant Bacteremia in Ventilated Patients: A Single-Center Experience in Korea. Acute Crit Care, 2018; 33(2): 73-82.

16. KOLLEF MH, et al. Appraising contemporary strategies to combat multidrug resistant gram-negative bacterial infections--proceedings and data from the Gram-Negative Resistance Summit. Clin Infect Dis., 2011; 53(2): 33-55.

17. KRAKER ME, et al. Will 10 Million People Die a Year due to Antimicrobial Resistance by 2050? PLoS Med., 2016; 13(11): e1002184.

18. LIU P, et al. Risk Factors for Carbapenem-Resistant Klebsiella pneumoniae Infection: A Meta-Analysis. Microb Drug Resist., 2018; 24(2):190-198.

19. MACVANE SH. Antimicrobial Resistance in the Intensive Care Unit: A Focus on Gram-Negative Bacterial Infections. J Intensive Care Med., 2017; 32(1): 25-3.

20. MAGILL SS, et al. Multistate point-prevalence survey of health care-associated infections. N Engl J Med., 2014; 370(13): 1198-208.

21. MATOS ECO, et al. Mortality in patients with multidrug-resistant Pseudomonas aeruginosa infections: a meta-analysis. Rev. Soc. Bras. Med. Trop., 2018; 51(4): 415-420.

22. MORRIS S, CERCEO E. Trends, Epidemiology, and Management of Multi-Drug Resistant Gram-Negative Bacterial Infections in the Hospitalized Setting. Antibiotics, 2020; 9(196): 1-20.

23. OSTHOFF M, et al. Urinary tract infections due to extended-spectrum beta-lactamase-producing Gram-negative bacteria: identification of risk factors and outcome predictors in an Australian tertiary referral hospital. Int J Infect Dis., 2015; 34: 79-83.

24. PATOLIA S, et al. Risk factors and outcomes for multidrug-resistant Gram-negative bacilli bacteremia. Ther Adv Infect Dis., 2018; 5(1): 11-18.

25. PILTCHER OB, et al. Como evitar o uso inadequado de antibióticos nas infecções de vias aéreas superiores? Posição de um painel de especialistas. Braz. j. otorhinolaryngol.,2018; 84(3): 265-279.

26. PULCINI C, et al. Design of a 'day 3 bundle' to improve the reassessment of inpatient empirical antibiotic prescriptions. J Antimicrob Chemother, 2008; 61(6): 1384-8.

27. RAMAN G, et al. Appropriate initial antibiotic therapy in hospitalized patients with gram-negative infections: systematic review and meta-analysis. BMC Infect Dis., 2015; 30(15): 395-406.

28. SALDANHA DMS, et al. O Uso Indiscriminado Dos Antibióticos: Uma Abordagem Narrativa Da Literatura. Revista Interfaces da Saúde, 2018; 5(1): 12-37.

29. SANDOVAL P, CHANG A. Factores de riesgo en infecciones por organismos multidroga resistentes en pacientes pediátricos oncológicos en la Unidad Nacional de Oncología Pediátrica (UNOP) de Guatemala. Rev. Fac. Med. (Guatemala), 2017; 1(22): 24-29.

30. SANTORO A, et al. Epidemiology and Risk Factors Associated With Mortality in Consecutive Patients With Bacterial Bloodstream Infection: Impact of MDR and XDR Bacteria. Open Forum Infect Dis., 2020; 7(11): ofaa461.

31. SILVA RA, et al. Resistência a Antimicrobianos: a formulação da resposta no âmbito da saúde global. Saúde em Debate, 2020; 44(126): 607-623.

32. TAVARES W. Antibióticos e quimioterápicos para o clínico. 4a ed. São Paulo: Atheneu, 2020; 824p.

33. TEDJA R, et al. The impact of multidrug resistance on outcomes in ventilator-associated pneumonia. Am J Infect Control., 2014; 42(5): 542-5.

34. UC-CACHÓN AH, et al. High Prevalence of Antimicrobial Resistance Among Gram-Negative Isolated Bacilli in Intensive Care Units at a Tertiary-Care Hospital in Yucatán Mexico. Medicina (Kaunas), 2019; 55(9): 1-13.

35. VIVAS R, et al. Multidrug-Resistant Bacteria and Alternative Methods to Control Them: An Overview. Microb Drug Resist., 2019; 25(6): 890-908.

36. WATTAL C, et al. Neonatal Sepsis: Mortality and Morbidity in Neonatal Sepsis due to Multidrug-Resistant (MDR) Organisms: Part 1. Indian J Pediatr., 2020; 87(2): 117-121. 